\title{
Electronic structure of silicon carbide polytypes studied by soft x-ray spectroscopy
}

\author{
J. Lüning, ${ }^{*}$ S. Eisebitt, J.-E. Rubensson, ${ }^{\dagger}$ C. Ellmers, and W. Eberhardt \\ Institut für Festkörperforschung, Forschungszentrum Jülich, D-52425 Jülich, Germany
}

(Received 15 January 1998; revised manuscript received 23 December 1998)

\begin{abstract}
The electronic structure of SiC polytypes is investigated with soft x-ray absorption (SXA) and emission (SXE) spectroscopy studying the local $\mathrm{C} p$ and $\mathrm{Si}(s+d)$ partial density of states (LPDOS) of $n$-doped cubic $3 \mathrm{C}$-SiC and hexagonal $4 \mathrm{H}$ - and $6 \mathrm{H}$-SiC. The shape and the energetic position of the occupied LPDOS of the valence band measured by nonresonantly excited SXE spectroscopy is nearly identical for the three polytypes, reflecting the local identity of the crystalline structures. The variation of the band gap from $2.2 \mathrm{eV}$ for $3 C$-SiC to $3.0 \mathrm{eV}$ for $6 H-\mathrm{SiC}$, and $3.3 \mathrm{eV}$ for $4 H-\mathrm{SiC}$ is caused by changes of the conduction band alone as reflected by the unoccupied LPDOS measured by SXA. Additionally, by resonantly excited SXE information on the band dispersion and the local symmetry character of the valence states is obtained. [S0163-1829(99)09715-5]
\end{abstract}

\section{INTRODUCTION}

The semiconducting indirect band gap material $\mathrm{SiC}$ has a large potential for applications in high power electronics ${ }^{1}$ due to its high stability, chemical inertness, and excellent heat conductivity [additional interest in $\mathrm{SiC}$ follows currently from the blue light emitting GaN diodes which can be grown on hexagonal SiC (Ref. 2)]. The crystal structure of SiC exhibits polytypism: Locally, all polytypes have the same diamondlike structure with each atom having four tetrahedrally coordinated neighbors of the other species. By stacking layers of such tetrahedra onto each other one achieves the crystal structure of cubic SiC (illustrated in Ref. 3) in which every fourth layer is identical, and this structure is named $3 C$-SiC. ${ }^{4}$ All other polytypes can be built by rotating some of the layers of the tetrahedra by $60^{\circ}$ relative to each other. Thus, arbitrary stacking series can be constructed which have mostly hexagonal crystal structures (some are rhombohedral). These are denoted as $n \mathrm{H}-\mathrm{SiC}$ (or $n \mathrm{R}-\mathrm{SiC}$ ) where $n$ is the number of layers in the stacking series. Stacking series of more than 100 layers have been observed, and it has recently also become possible to grow several polytypes as single crystals. Earlier, only two phases of $\mathrm{SiC}$ were distinguished, namely, the cubic phase referred to as $\beta$-SiC, and everything else referred to as (different types of) $\alpha$-SiC. One can attribute a "hexagonal character' to the crystal structure of the $\mathrm{SiC}$ polytypes ${ }^{5}$ which gives the fraction of "hexagonal tetrahedron layers" (two identical neighbor layers) in contrast to "cubic layers" (two different neighbor layers) in the crystal unit cell.

All SiC polytypes thus have the same nearest neighbor configuration while they differ in their long range order, which influences the electronic structure significantly. For example, the band gap varies from $2.2 \mathrm{eV}$ for $3 C$-SiC to 3.0 $\mathrm{eV}$ for $6 H-\mathrm{SiC}, 3.3 \mathrm{eV}$ for $4 H-\mathrm{SiC}$, and $3.5 \mathrm{eV}$ for $2 H-\mathrm{SiC}{ }^{6}$ i.e., the band gap increases with increasing hexagonal character of the polytype. Hence, it is possible to tune the band gap of the material within this energy range, if one can control the stacking order in the growth process. It is worth noting that it is the electronic structure which transfers the information about the stacking order to the surface during the growth process.
We investigated the electronic structure of cubic $3 C$-SiC and hexagonal $4 H$ - and $6 H$-SiC by synchrotron radiation excited soft X-ray emission (SXE) and absorption (SXA) spectroscopy. For nonresonant excitation energies SXE spectra map the local partial density of states. These results are discussed in Sec. III. When the energy of the synchrotron radiation used for excitation is well defined and it is tuned to an absorption threshold (or slightly above) it becomes possible to deduce band structure information from the spectra. This gives the unique opportunity to perform local symmetry resolved band mapping. We discuss these results in Sec. IV.

The electronic structure of cubic $\mathrm{SiC}$ has been investigated with nonresonantly excited SXE before. The highenergy excited $\mathrm{Si} L_{2,3}$ SXE spectra shown here are in good agreement with previous studies ${ }^{7,8}$ which were notably resolution limited. The C $K$ SXE has been measured earlier for cubic SiC, ${ }^{9}$ showing a similar shape but being slightly broadened compared to the results presented here. For the hexagonal polytypes to our knowledge no SXE data exist in the literature so far for distinct polytypes [even though $\mathrm{Si} L_{2,3}$ SXE spectra of (uncharacterized) $\alpha$-SiC have been reported $\left.^{8}\right]$.

\section{EXPERIMENT}

Experiments were carried out at the undulator beamline BW3 at HASYLAB, Germany. ${ }^{10}$ The absolute energy scale of the monochromator was calibrated at the $\mathrm{Si} L_{3}$ absorption threshold. ${ }^{11}$ All SXE spectra were recorded with a Rowlandtype spectrometer built in our institute. For the $\mathrm{Si} L_{2,3} \mathrm{SXE}$ spectra a grating of $3.71 \mathrm{~m}$ with 600 lines $/ \mathrm{mm}$ was used, resulting in an experimentally determined resolution of 200 meV when employing a $30 \mu \mathrm{m}$ entrance slit. With the same entrance slit but a grating of $5 \mathrm{~m}$ radius and 1200 lines $/ \mathrm{mm}$ a resolution of $400 \mathrm{meV}$ was achieved for the $\mathrm{C} K$ spectra. The energy scale of the SXE spectra was established by reflection of monochromatized synchrotron radiation into the spectrometer calibrating the SXE and the SXA energy scales relative to each other.

For the nonresonantly excited $\mathrm{Si} L_{2,3}$ and $\mathrm{C} K$ spectra discussed in Sec. III photon energies well above the absorption thresholds were chosen, i.e., in the case of the $\mathrm{Si} L_{2,3}(\mathrm{C}$ 
$K)$ threshold $148 \mathrm{eV}(295 \mathrm{eV})$. The excitation energy bandwidth of the monochromator was set for all excitation energies above the Si $L_{2,3}(\mathrm{C} K$ ) thresholds to be $50 \mathrm{meV}$ (260 $\mathrm{meV}$ ).

In soft x-ray absorption spectroscopy the energy of the incoming photons is scanned and the absorption cross section is measured. In the soft x-ray energy range this is generally done using yield techniques which monitor the number of secondary decays following a core level excitation, i.e., electron or photon emission due to Auger or fluorescence decay of the core hole state, respectively. ${ }^{12}$

In order to be bulk sensitive the $\mathrm{Si} L_{2,3}$ and $\mathrm{C} K$ SXA spectra were recorded by monitoring the fluorescence yield (FY) with a channeltron. Saturation due to the lack of selfabsorption in FY detection of SXA spectra ${ }^{13}$ was avoided by choosing a normal-in grazing-out geometry. The bandwidth of the monochromator was set for the SXA spectra at the $\mathrm{Si}$ $L_{2,3}(\mathrm{C} K)$ edge to $10 \mathrm{meV}(50 \mathrm{meV})$.

The $\mathrm{SiC}$ samples are commercially grown single crystals. ${ }^{6}$ $3 C$-SiC was grown on a silicon single crystal wafer, while the hexagonal single crystals were grown on polycrystalline substrates of the respective material. All samples are $n$-doped semiconductors using nitrogen with a density of 2 $\times 10^{16} / \mathrm{cm}^{3} 3\left(\mathrm{C}\right.$-SiC), $7 \times 10^{17} / \mathrm{cm}^{3} 6(H-\mathrm{SiC})$, respectively $4 \times 10^{18} / \mathrm{cm}^{3} 4(H-\mathrm{SiC})$. Contribution of the substrate to the spectra can be excluded as all samples were an order of magnitude thicker than the attenuation length of the soft $\mathrm{x}$-ray photons.

\section{LOCAL PARTIAL DENSITY OF STATES (LPDOS)}

\section{A. Nonresonantly excited SXE and LPDOS}

The most direct approach to investigate the variation of the valence band (VB) electronic structure with the stacking series would be photoemission spectroscopy (PES). Due to the short mean free path of electrons, however, PES is a surface sensitive technique, and it is in general difficult to apply this electron spectroscopy technique to (large band gap) semiconductors because of charging effects. Nevertheless, for cubic $\mathrm{SiC}$ the top of the VB was mapped close to the $\Gamma$ point along the $\Delta$ direction with angle resolved PES. ${ }^{14}$ The interpretation of SiC PES spectra is further complicated by the existence of a large variety of surface reconstructions depending on the method of sample preparation. ${ }^{15}$

These impediments can be overcome by studying the VB electronic structure using soft x-ray emission spectroscopy. In SXE spectroscopy the energy of emitted photons is analyzed which results from the radiative decay of a shallow core electron vacancy involving the transition of a VB electron to the core state. The transition probability is to a good approximation proportional to the VB density of states (DOS), more precisely, to the local partial density of states: ${ }^{16}$ Due to the spatial restriction of core wave functions and the dipole character of radiative transitions in the soft $\mathrm{x}$-ray range the spectral distribution of the emitted photons is determined by the DOS projected to the atomic site (local) and the symmetry corresponding to the well-defined angular momentum of the involved core state (partial). The energy dependence of the transition matrix element itself is generally assumed to be small compared to the DOS changes in

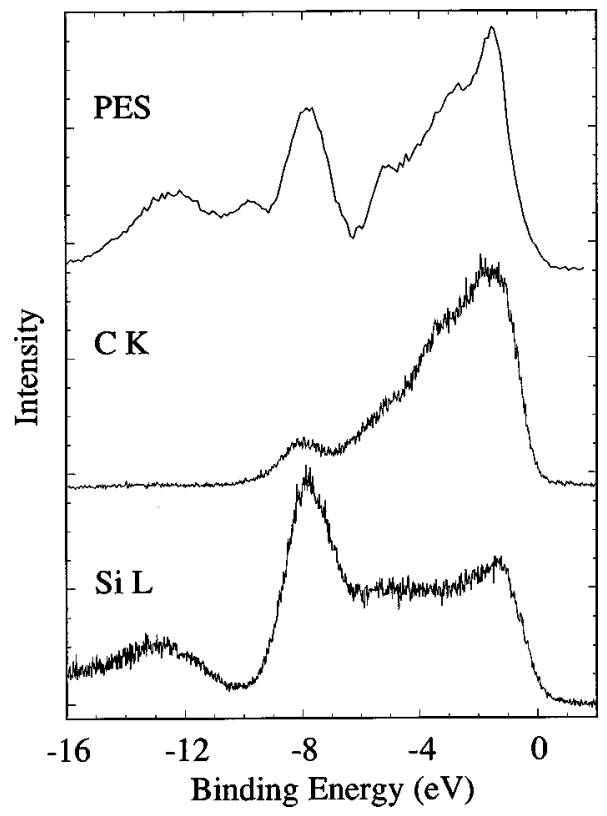

FIG. 1. Comparison of $\mathrm{C} K$ and $\mathrm{Si} L_{2,3} \mathrm{SXE}$ spectra for $3 C$-SiC with the VB PES spectrum (Ref. 18). All spectra are referred to a common binding energy scale as explained in the text.

the energy range of interest. Furthermore, it is the DOS of the final VB hole state which is mapped in SXE spectroscopy according to the final state rule, ${ }^{17}$ i.e., SXE probes the same electronic structure as VB PES does.

The SXE spectra are in this approximation independent of the method of excitation of the core hole vacancy. Hence, the complete process can be described within the intuitive twostep "excitation-followed-by-emission" picture. Highenergy electron bombardment or nonresonant photon excitation (with energies well above the absorption threshold of interest) are generally used for core hole excitation.

\section{B. Occupied LPDOS of cubic SiC}

The benefits of the selection rules in SXE spectroscopy are demonstrated in Fig. 1 where the Si $L_{2,3}$ and $\mathrm{C} K$ SXE spectra are compared to a VB PES spectrum. ${ }^{18}$ The initial core hole states for the SXE spectra were created by photon excitation with photon energies well above the binding energy of the core electron of interest. All spectra are referred to a common binding energy scale. This is done by subtracting from the energy scale of each spectrum the energy of the high-energy cutoff which corresponds to the VBM (as determined by linear extrapolation, see Fig. 3 and Fig. 4).

The PES spectrum plotted at the top of Fig. 1 is dominated by three emission peaks centered at $-1.5 \mathrm{eV},-8.0$ $\mathrm{eV}$, and at $-12.4 \mathrm{eV}$ which are accompanied by a weaker line around $-9.9 \mathrm{eV}$.

The spectrum in the middle is the C $K$ SXE spectrum associated with the fluorescence decay of a $\mathrm{C} 1 s$ core hole. Similar to the photoelectron spectrum the $\mathrm{C} K$ spectrum shows a sharp rise at the VB maximum (VBM) peaking at $-1.5 \mathrm{eV}$. Towards the bottom of the VB the emission intensity decreases continuously with a weak shoulder around $-3.5 \mathrm{eV}$ ending in a weak peak at $-8.0 \mathrm{eV}$. This indicates that the electronic states of local $p$ symmetry at the $\mathrm{C}$ sites concentrate in the upper part of the VB. 


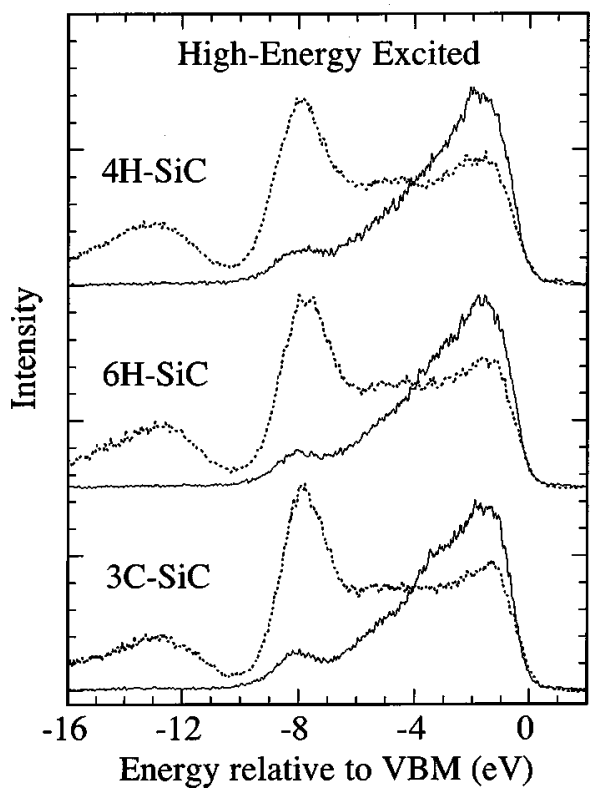

FIG. 2. Comparison of $\mathrm{C} K$ (solid lines) and $\mathrm{Si} L_{2,3}$ (dashed lines) SXE spectra for $3 C-, 6 H-$, and $4 H-S i C$. Photons with energy well above the absorption threshold (149 eV and $302 \mathrm{eV}$ for Si $L_{2,3}$ and $\mathrm{C} K$, respectively) were used for excitation. The common binding energy scale is established as described in the text.

At the bottom of the figure the Si $L_{2,3}$ SXE spectrum is given. It is dominated by a peak around $-8.0 \mathrm{eV}$ which develops into a flat plateau ranging up to the VBM where it drops sharply. On the low-energy side of the main peak one finds around $-13 \mathrm{eV}$ a separate broad feature. This peak corresponds to a second lower VB (LVB) split by a gap of around $2 \mathrm{eV}$ from the main upper VB (UVB) (compare, e.g., the calculations reproduced in Ref. 20). Hence, states of local

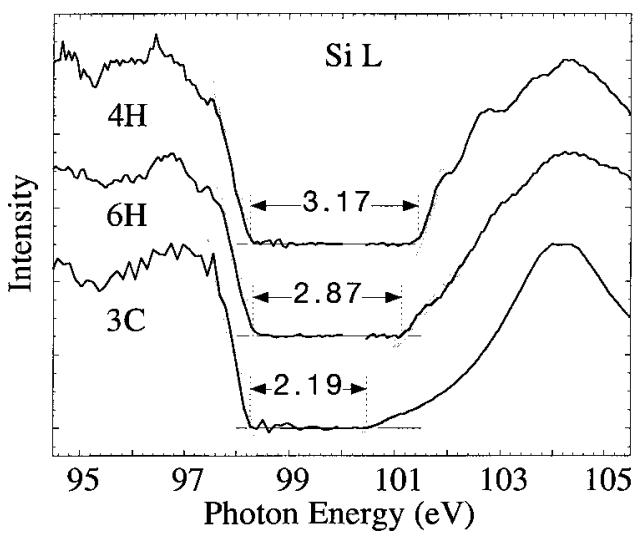

FIG. 3. Si $L_{3}$ SXE and $L_{2,3}$ SXA spectra in the vicinity of the band gap (normalized to same maximal intensity) of $3 \mathrm{C}-, 6 \mathrm{H}-$, and $4 H$-SiC. The Si $L_{3}$ SXE spectra are obtained by tuning the photon excitation energy to be below the $L_{2}$ absorption threshold (the spinorbit splitting is $0.6 \mathrm{eV}$ ). In this context it is important to note that the spectral shape close to the top of the VB does not depend on the excitation energy (see Figs. 6, 8, and Fig. 2 in Ref. 20). The energy of the VBM and the CBM are determined by linear extrapolation of the edges to the baseline as indicated by the gray bars. The band edge positions are listed in Table I. The deduced band gaps are in good agreement with the literature values measured by optical absorption.

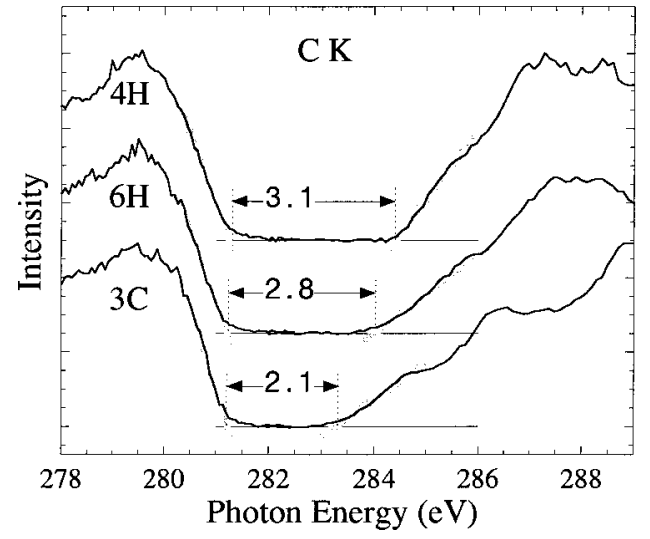

FIG. 4. Same as Fig. 3 but for the $C K$ edge. The energy positions of VBM and CBM are listed in Table II. The deduced band gaps are in good agreement with the literature values measured by optical absorption and the values found at the $\mathrm{Si} L_{3}$ edge.

$\mathrm{Si}(s+d)$ symmetry dominate at the bottom of the UVB but can be also be found throughout the whole VB region.

As the excitation energy is chosen well above the $\mathrm{Si} L_{3}$ and $L_{2}$ absorption threshold electrons from both spin-orbit split $2 p$ levels can be excited. The spectrum is therefore a superposition of the local DOS with itself, shifted by the spin-orbit splitting of $0.6 \mathrm{eV}$ and with the relative intensities of $2: 1$ corresponding to the statistical population of the sublevels (neglecting correlation effects which are generally weak in broadband materials). This superposition is reflected by the weak shoulder around $-7.4 \mathrm{eV}$ and the small foothill close to the high-energy cutoff which are the $L_{2}$ equivalents of the main peak at $-8.0 \mathrm{eV}$ and the VBM associated with core holes in the $L_{3}$ level, respectively. This has, however, only a minor influence on the overall shape of the spectrum as the main features are much broader than the spin-orbit splitting.

Comparing the $\mathrm{C} K$ and $\mathrm{Si} L_{2,3} \mathrm{SXE}$ spectra to each other one notices that (with one exception) the strong peaks of one SXE spectrum also appear as weaker features in the other. This shows that the VB states do not have pure local symmetries but that the atomic states are hybridized due to the influence of the solid state environment.

The exception is the peak around $-13 \mathrm{eV}$ in the $\mathrm{Si} L_{2,3}$ SXE spectrum which does not have any equivalent feature in the $\mathrm{C} K$ spectrum. This indicates that the states forming the LVB (which derive from the atomic C $2 s$ orbitals) must have (nearly) pure local $s$ symmetry at the $\mathrm{C}$ site. This is in contrast to the local symmetry of these states at the Si sites. The Si $K_{\beta}$ spectrum of Wiech ${ }^{7}$ reveals that states of the LVB also have local Si $p$ symmetry.

Using the SXE spectra one can assign the emission lines of the VB photoelectron spectrum. Hence, the peak just below the VBM at $-1.5 \mathrm{eV}$ is due to band states with local $p$ symmetry at the carbon sites while the two other strong peaks at deeper binding energies $(-8.0 \mathrm{eV}$ and $-12.4 \mathrm{eV})$ belong to states with local $(s+d)$ symmetry at the silicon sites.

Furthermore, the comparison reveals that the weak emission feature at $-9.9 \mathrm{eV}$ in the PES spectrum does not have one of these local symmetries. Even more, one may doubt that this peak is due to a DOS maximum of bulk $\mathrm{SiC}$ at all as 
TABLE I. Comparison of band gaps derived by soft x-ray spectroscopy (SXS) from Si $L_{3}$ SXE and SXA in Fig. 3 with the values from optical spectroscopy (Ref. 6) on samples prepared in the same way. The uncertainty of the VBM and CBM energies relative to each other is around $\pm 0.05 \mathrm{eV}$. All values are given in $\mathrm{eV}$.

\begin{tabular}{lcccc}
\hline \hline & VBM & CBM & SXS gap & Optical gap \\
\hline $3 C$ & 98.31 & 100.50 & $2.2 \pm 0.1$ & 2.2 \\
$6 H$ & 98.28 & 101.15 & $2.9 \pm 0.1$ & 3.0 \\
$4 H$ & 98.27 & 101.44 & $3.2 \pm 0.1$ & 3.3 \\
\hline \hline
\end{tabular}

it is located just in the gap between the LVB and the UVB. Having the surface sensitivity of PES in mind we therefore assign this line to originate from the sample surface, i.e., from surface states or a contamination of the surface.

\section{Occupied LPDOS of hexagonal polytypes}

In Fig. 2 we compare the $\mathrm{C} K$ (solid lines) and the $\mathrm{Si} L_{2,3}$ (dashed lines) SXE spectra of the three different polytypes, i.e., the cubic polytype $3 \mathrm{C}$ and the hexagonal polytypes $4 H$ and $6 H$. For this comparison the spectra are again referred to a common binding energy scale by aligning the VBM (see Fig. 3 and Fig. 4). One notices a strong similarity between the spectra of the different polytypes, reflecting the local identity of the crystal structures. The influence of the different long range order can only be found in minor details such as the width of the dominant peak at $-8.0 \mathrm{eV}$ in the Si $L_{2,3}$ spectra or its intensity relative to the plateau. The similarity of the spectra is evidence for the fact that the occupied LPDOS is almost unaffected by the stacking order. The stacking order, however, does influence the crystal momentum of the band states as we will demonstrate below.

Apart from the agreement in shape we also observe that the VBM energies in the Si $L_{2,3}(\mathrm{C} K)$ spectra of the different polytypes coincide within the experimental uncertainty of $\pm 0.05 \mathrm{eV}( \pm 0.1 \mathrm{eV})$. This is demonstrated in Fig. 3 and Fig. 4 for the $\mathrm{Si} L_{3}$ and $\mathrm{C} K$ edge, respectively, where the emission energies of the VBM are determined by extrapolation of the high-energy cutoff in the $\mathrm{Si} L_{3}$ and $\mathrm{C} K \mathrm{SXE}$ spectra. The values derived in this way are listed in Tables I and II, and were used to establish the common energy scales in Fig. 1 and Fig. 2.

We would like to remind the reader that the energy scales in SXE and SXA spectroscopy are relative to the binding energy of the involved core level. As these binding energies may be subject to chemical shifts ${ }^{21}$ the SXE spectra do not give the position of the VBM on an absolute energy scale, e.g., relative to the Fermi energy. We expect, however, that

TABLE II. Same as table I but for $\mathrm{C} K$ edge, values derived from Fig. 4 are given in eV. The uncertainty of the VBM and CBM energies relative to each other is around $\pm 0.10 \mathrm{eV}$. The higher uncertainty is caused by the lower resolution at the $\mathrm{C} K$ edge.

\begin{tabular}{lcccc}
\hline \hline & VBM & CBM & SXS gap & Optical gap \\
\hline $3 C$ & 281.19 & 283.31 & $2.1 \pm 0.2$ & 2.2 \\
$6 H$ & 281.22 & 284.02 & $2.8 \pm 0.2$ & 3.0 \\
$4 H$ & 281.25 & 284.39 & $3.1 \pm 0.2$ & 3.3 \\
\hline \hline
\end{tabular}

differences in the chemical shift of the core level binding energies are negligible as the main factors which influence core level binding energies are similar for the polytypes. First, the nearest neighbor atom types and positions, which dominate the electrostatic influence on the binding energy, are identical in the polytypes. Second, the core hole screening capability can be expected to be comparable due to the similar bandwidths in the polytypes.

Core level binding energies can be determined relative to the Fermi energy by x-ray photoelectron spectroscopy (XPS). ${ }^{22}$ As expected from the above discussion we have found the same binding energies for these particular $n$-doped $\mathrm{SiC}$ samples. We note, however, that doping density and band bending at the surface may affect the XPS data.

\section{Unoccupied LPDOS of SiC polytypes}

Both the similar shape of the VB and the same emission energy location of the VBM for the three polytypes are surprising results, given the variation of the band gap from 2.2 $\mathrm{eV}$ for $3 C-\mathrm{SiC}$ to $3.3 \mathrm{eV}$ for $4 H-\mathrm{SiC}$. These observations imply that the strong variation of the band gap size originates from changes in the conduction band (CB). This assumption can be verified experimentally by SXA spectroscopy, which can be used to determine the energy difference between a core level and the $\mathrm{CB}$ minimum (CBM).

The Si $L_{2,3}$ SXA spectra in the vicinity of the band gap are shown for the three different polytypes on the right side in Fig. 3. Comparing the onset of the absorption in the SXA spectra one finds, as expected, that the CBM shifts to higher energies by the amount of the increase of the optical gap. This shift is accompanied by an increase of the slope at the onset of the absorption suggesting that the empty states are "pushed" towards higher energies, resulting in a "pile up", of the DOS close to the CBM. Even for energies higher above the CBM the SXA spectra show differences indicating that the unoccupied DOS is different for the three polytypes not only close to the CBM. Note that the sharpening of the $\mathrm{CB}$ edge with increasing band gap, in contrast to the similarity of the VB edge, supports the result discussed above, i.e., that the gap opening originates mainly from changes in the unoccupied states.

Within the DOS interpretation of SXE and SXA the energy distance of VBM and CBM must equal the band gap of the material. This interpretation neglects correlation effects, in particular, the Coulomb interaction between the (localized) positive core hole and the excited electron referred to as (core) excitonic effect. Therefore, the gap value as determined by SXE and SXA in comparison with the band gap (e.g., from optical absorption) can be used to estimate the importance of excitonic effects. The values derived from Fig. 3 are compared to the optical band gaps from Ref. 6 in Table I. We find good agreement, demonstrating that excitonic effects play only a minor role at the $\mathrm{Si} L_{3}$ edge in the $\mathrm{SiC}$ polytypes. This is understandable as the screening of the positive core hole in the final state of the absorption by the VB electrons should be rather effective due to the large width of the VB.

The near band gap region of the $\mathrm{C} K$ spectra is shown in Fig. 4. One notices the same behavior of the VB and the $\mathrm{CB}$ as in the $\mathrm{Si} L_{2,3}$ spectra, i.e., the band gap increases due to a shift of the CBM to higher binding energy which is accom- 
panied by an increased slope of the bottom of the CB. The VBM and the CBM positions as well as the band gaps derived from the spectra in Fig. 4 are listed in Table II.

Comparing the gap values determined at the $\mathrm{C} K$ edge to the values following from the spectra at the $\mathrm{Si} L_{3}$ edge one finds good agreement within the experimental uncertainty. This indicates that the states forming VBM and CBM have a nonvanishing amplitude at both atomic sites due to hybridization.

The uncertainty in the determination of the band gaps is larger at the $\mathrm{C} K$ edge as a result of the lower resolution in these spectra. This lower resolution also makes it reasonable that the gaps determined at the $\mathrm{C} K$ edge are systematically smaller than the ones resulting from the $\mathrm{Si} L_{3}$ edge data. The distance of the VBM and CBM as determined here corresponds only to the band gap if the slope of the DOS is smaller than the (lifetime and resolution) broadening present in the spectra. In this case the convolution of the DOS by the broadening functions is negligible, and the extrapolation yields the DOS onset. If, on the other hand, the broadening becomes comparable to the slope of the DOS, the convolution gives rise to a shift of the DOS onset which lowers the derived band gap. This may be the case for the resolution of only $0.4 \mathrm{eV}$ at the $\mathrm{C} K$ edge.

We note that the shape of the C $K$ SXA spectra may be influenced by surface contaminations with carbon atoms. Although SXA as measured by FY is bulk sensitive the carbon contamination of optical beamline elements introduces artificial distortions in the spectra. These fluctuations were corrected by monitoring the incoming photon flux $I_{0}$ via the electron yield of a gold mesh introduced into the photon beam. As the mesh is likely to be carbon contaminated itself this correction involves some uncertainties. The region close to the CBM, however, is unaffected as the $I_{0}$ signal was without any structure in this region.

Park et al. ${ }^{23}$ performed ab initio pseudopotential calculations within the local-density approximation for $3 \mathrm{C}-, 6 \mathrm{H}-$, and $4 \mathrm{H}$-SiC. In accordance with our experimental results the computed VB DOS of the various polytypes shows a strong similarity, while the unoccupied DOS varies with the polytypes. Especially, the sharpening of the DOS at the CB edge with increasing gap size is also present in the calculation. This behavior can also be inferred from the band structure calculations of Käckell, Wenzien, and Bechstedt ${ }^{24}$ reproduced for the hexagonal polytypes in Figs. 5-8.

\section{LOCAL SYMMETRY RESOLVED BAND MAPPING}

\section{A. Resonant inelastic soft x-ray scattering (RIXS) within a two-step picture}

So far we have treated the SXE process as independent of the preceding core hole excitation process. However, the way the core hole, required for the fluorescence decay, is prepared can have an influence on the SXE spectra. First of all, excitation by electron or ion bombardment has (apart from sample destruction) the disadvantage that the energy transfer in the excitation is not well defined. This generally gives rise to satellite structures in the spectra which result from the decay of excited states including additional excitations be-

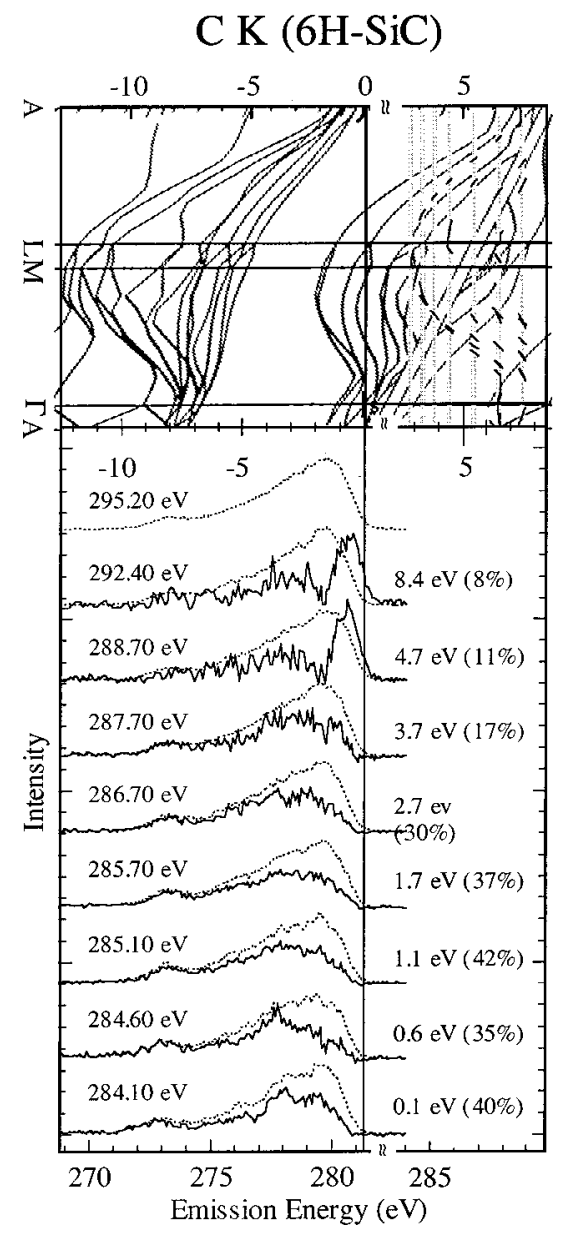

FIG. 5. Resonantly excited SXE spectra of $6 H$-SiC (dashed lines) normalized to the same area for excitation energies in the vicinity of the $\mathrm{C} K$ absorption threshold as indicated by the energy values on the left side. The high-energy excited spectrum at the top maps the $p$-symmetric DOS locally at the carbon sites. The solid lines show the normalized coherent scattering contributions. The energy values on the right side indicate the energy of the excited energy electron in the $\mathrm{CB}$ relative to the CBM. The percentage values quantify the coherent contribution to the measured spectrum. The dashed bars in the band structure calculation (Ref. 24) at the top of the figure indicate the energy of the core electron excited into the $\mathrm{CB}$ for the various excitation energies. The intersections with the unoccupied bands determine the CB states which can be populated at the corresponding excitation energy. The theoretical band gap is adjusted to the experimental value of $3.0 \mathrm{eV}$.

sides the core hole. The occurrence of such satellites reflects the evidence of correlation effects in the material under investigation. ${ }^{25}$

This problem can be overcome by using photon excitation with energies close to the absorption threshold of interest, thereby prohibiting additional excitations by the lack of energy surplus or the small probability for multiple excitation processes at the selected energy. Since the advent of high brilliance synchrotron sources their tunability has therefore been explored. We note in this context that the SXE spectra discussed in the preceding section are not affected by satellite structures, which is also due to the high efficiency of screening in $\mathrm{SiC}$. 


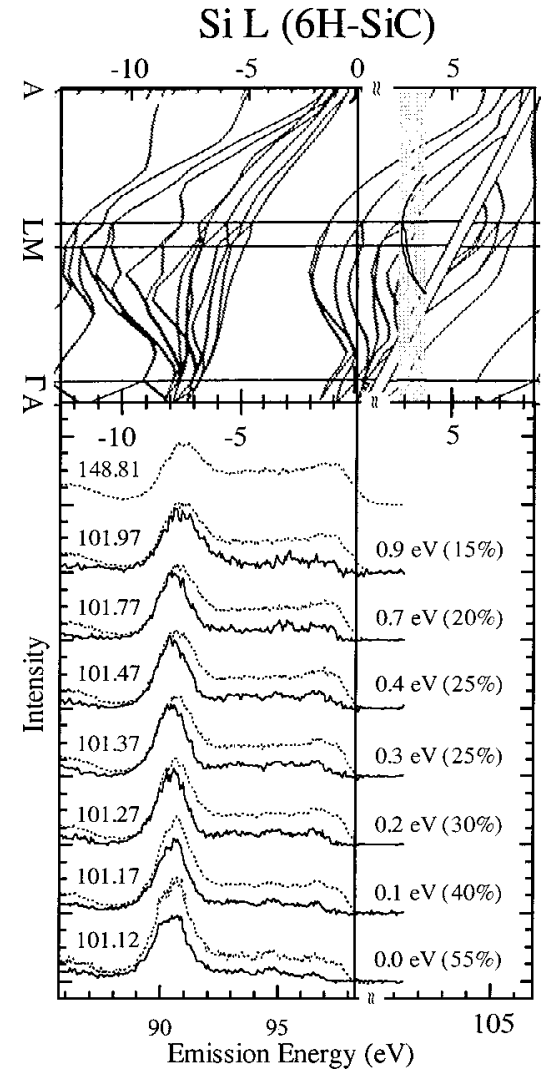

FIG. 6. Resonantly excited Si $L_{2,3}$ SXE spectra of $6 H$-SiC (dashed lines) and coherent scattering contributions (solid lines). The high-energy excited spectrum at the top maps the $(s+d)$-symmetric DOS locally at the silicon sites. For other details see caption of Fig. 5. Note that due to the spin-orbit splitting of the Si $2 p$ level the high-energy excited spectrum is a superposition of the LPDOS with itself shifted by the spin-orbit splitting and weighted by the population ratio of the sublevels. For the extraction of the coherent fraction for the two lower most spectra excited below the Si $L_{2}$ edge the LPDOS has to be extracted from the high-energy excited spectrum. This was done taking the spin-orbit splitting of $0.6 \mathrm{eV}$ into account and assuming an intensity ratio of $2: 1$ for $L_{3}: L_{2}$.

A further dependence of the SXE spectra of broadband materials on the energy of the photons used for core hole excitation was observed by Rubensson et al. ${ }^{26}$ in 1990 . Using monochromatized synchrotron radiation these authors studied the Si $L_{2,3}$ SXE spectra of crystalline Si for energies of the incident photons in the vicinity of the Si $2 p$ absorption thresholds. The observed energy dependence was explained two years later within the theory of "resonant inelastic soft x-ray scattering, ${ }^{, 27,28}$ and has been observed since then for a wide range of broadband materials (e.g., Refs. 20 and 2932).

The basic idea is that the SXA process preparing the core hole excitation and the "subsequent" SXE process cannot be treated as independent processes but must be regarded as a one-step scattering event when exciting resonantly, i.e., in the vicinity of an absorption threshold: The initial state of the entire process includes an incoming photon and the electronic ground state, while the final state consists of an outgoing photon and a VB to $\mathrm{CB}$ excitation in the electronic system. In this kind of process the total momentum is con-

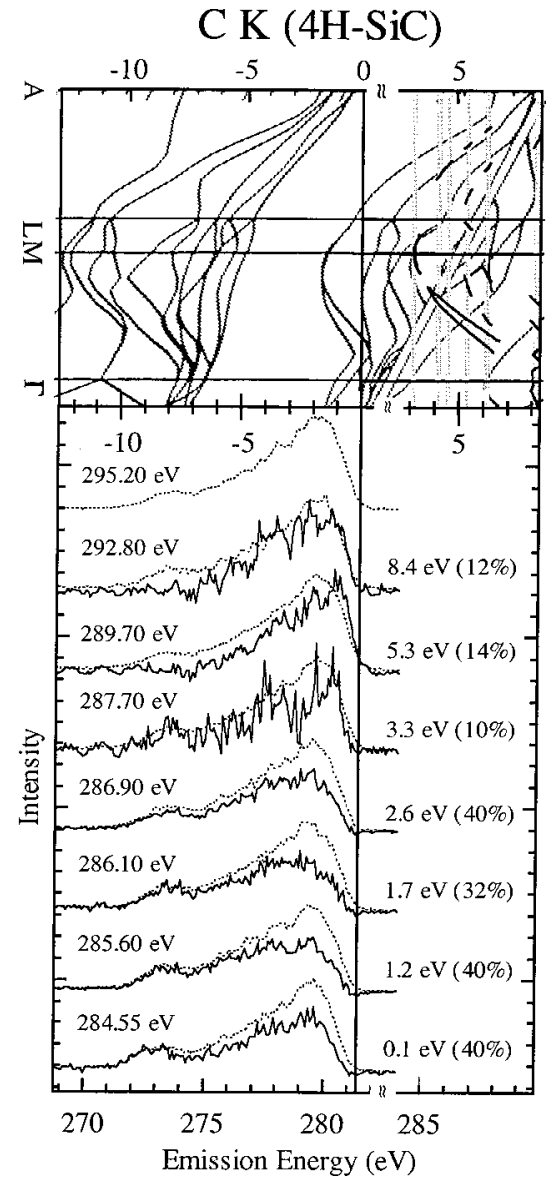

FIG. 7. Resonantly excited C $K$ SXE spectra (dashed lines) and coherent scattering contributions (solid lines) of $4 \mathrm{H}$-SiC. The band structure calculation at the top is reproduced from Käckel, Wenzien, and Bechstedt (Ref. 24). The theoretical band gap is adjusted to the experimental value of $3.2 \mathrm{eV}$. For other details see caption of Fig. 5.

served, i.e., the change of crystal momentum (modulo a reciprocal lattice vector) must be balanced by the momentum transfer of the scattered photon. The latter is in the soft x-ray range negligible in comparison with momenta of the electronic system. The momentum of the excited CB electron and of the VB hole in the final state must therefore be identical, which corresponds to a vertical excitation in a reduced band structure scheme. The excitation energy $E_{\text {exc }}$ compared to the core electron binding energy $E_{\text {bind }}$ determines in conjunction with the dispersion of the band structure $E(\mathbf{k})$ the momentum of the excited electron in the $\mathrm{CB}, \mathbf{k}_{e}$, as a consequence of energy conservation: $E\left(\mathbf{k}_{e}\right)=E_{\text {exc }}-E_{\text {bind }}$. As a result the momentum of the $\mathrm{VB}$ electron taking part in the scattering event can be controlled by the choice of the excitation energy $E_{\mathrm{exc}}$. This is the origin of the momentum selectivity necessary to obtain momentum selective band structure information. ${ }^{33}$

\section{B. Resonantly excited SXE spectra}

The dashed lines in Figs. 5-8 show the SXE spectra of the hexagonal $\mathrm{SiC}$ polytypes excited resonantly in the vicinity of the $\mathrm{C} K$ and the $\mathrm{Si} L_{2,3}$ threshold. The corresponding spectra series of $3 C-\mathrm{SiC}$ are published in Ref. 20. The exci- 


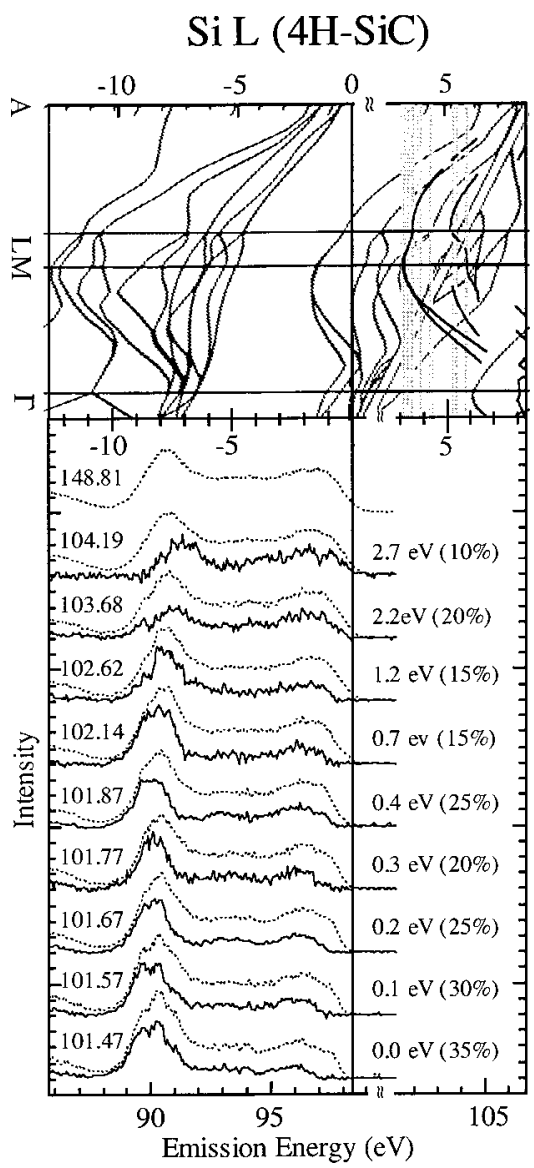

FIG. 8. Resonantly excited Si $L_{2,3}$ SXE spectra (dashed lines) and coherent scattering contributions (solid lines) of $4 \mathrm{H}$-SiC. The band structure calculation at the top is reproduced from Käckel, Wenzien, and Bechstedt (Ref. 24). The theoretical band gap is adjusted to the experimental value of $3.2 \mathrm{eV}$. For other details see caption of Fig. 6.

tation energy (given on the left side of the spectra) increases from "at threshold" for the spectra plotted at the bottom to "well above" for the one at the top (shown previously in Fig. 2). The energy values on the right side of the spectra indicate the energy of the excited electron relative to the CBM. Above the spectra we reproduce a band structure calculation adapted from the literature. ${ }^{24}$

One notes clearly that the shape of the SXE spectra changes with the excitation energy. These changes, however, are not as distinct as expected from the theoretical considerations made above. The reason for this is still under debate, ${ }^{34}$ and we discussed this topic in detail in a previous publication $^{20}$ concentrating on the methodical aspects of RIXS. Here we only recapitulate the result of our previous paper which follows the arguments of Refs. 27 and 28. Thus, the relation between the momenta of the excited CB electron and the VB hole is only maintained if no other momentum carrying particle is involved in the scattering event. In a solid, however, there is the possibility of electron-phonon and electron-electron scattering whereby momentum is transferred to a particle that is not detected in this experiment. If such scattering processes occur, the relation between the momentum of the excited CB electron and of the resulting VB hole breaks down. This leads to a contribution to the measured spectrum from states of a wide range of the Brillouin zone (BZ). This contribution has been named the incoherent fraction in the original RIXS paper to indicate that not all particles contributing to the final state are observed. Consequently, the scattering fraction with only the single VB to $\mathrm{CB}$ excitation in the final state is referred to as coherent fraction (this nomenclature might be misleading as coherence in the sense of the ability for interference is of no relevance in this context).

The coherent fraction from the spectra is extracted assuming that in the ensemble of decays leading to the incoherent fraction the momentum relation has broken down completely. ${ }^{35}$ In this case the incoherent fraction is given by the LPDOS as measured in nonresonantly excited SXE spectroscopy and the coherent fraction can be determined by simply subtracting the LPDOS from the resonantly excited spectrum under the restriction that the intensity of the difference spectrum remains positive. It is of importance to note that within the RIXS interpretation this subtraction procedure is unambiguous. The restriction of a positive difference gives a well-defined upper limit for the subtraction. Subtracting less, on the other hand, would imply that a part of the coherent fraction is identical to the LPDOS, which cannot be the case as only a fraction of the states in the BZ is allowed to contribute to the scattering process.

The coherent fractions resulting from these subtraction procedures are indicated by the solid lines in Figs. 5-8, and their contribution to the measured RIXS spectra is given by the percentage values on the right side of the spectra.

The coherent fractions thus extracted change in a complex manner. Apart from peaks shifting with excitation energy (e.g., the dominant peak around $-8 \mathrm{eV}$ in the $\mathrm{Si}$ spectra) one also observes new emission features (e.g., in the $\mathrm{C}$ spectra excited at higher energies). These changes not only occur close to threshold but are found for higher excitation energies, too.

\section{Band dispersion of cubic SiC}

We start the detailed analysis of the coherent fractions with the threshold excited spectra of cubic $\mathrm{SiC}$ plotted at the bottom of Fig. 9. Both spectra are dominated by a single line which in the case of the $\mathrm{C}$ and Si threshold spectra is located at $-3.5 \mathrm{eV}$ and at $-8.0 \mathrm{eV}$, respectively.

In our previous publication ${ }^{20}$ we demonstrated how quantitative band structure information can be gained from these spectra, and we refer to this reference for that purpose. Here we only point out that the states below $-10 \mathrm{eV}$ of the LVB contribute to neither of the coherent threshold spectra. As they do not contribute to the $\mathrm{C} K$ nonresonantly excited spectrum it is expected that they do not contribute to the spectrum excited at the $\mathrm{C} K$ threshold. The absence in the threshold excited $\mathrm{Si} L_{3}$ scattering spectrum, on the other hand, indicates that not all states of the LVB have local $\mathrm{Si}(s$ $+d$ ) symmetry (as the states at the $X$ point do not). We further mention that for higher excitation energies in some of the Si spectra shown in Ref. 20 one finds a weak contribution in the energy region of the LVB (e.g., in the one excited at $103.18 \mathrm{eV}$ ). This indicates that for the crystal momenta involved in the scattering for those excitation energies states with local $\mathrm{Si}(s+d)$ symmetry exist in the LVB. As with increasing excitation energy states further away from the $X$ 


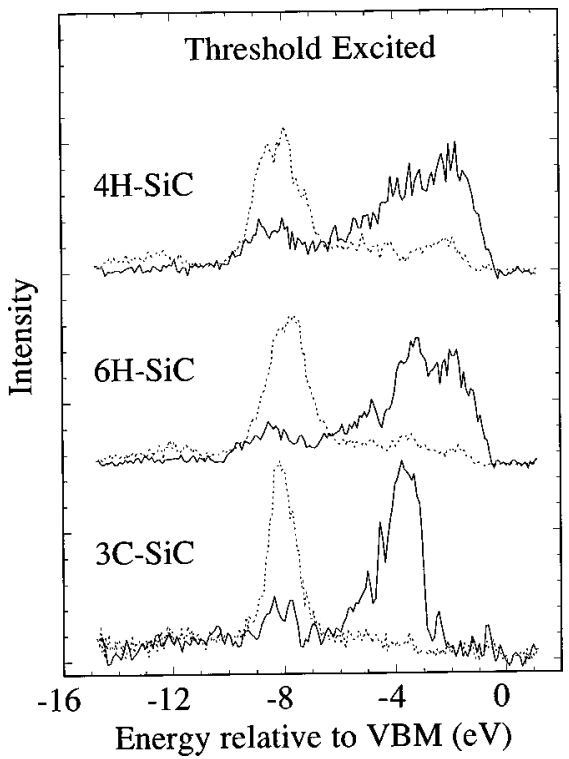

FIG. 9. Comparison of the threshold excited C $K$ (solid lines) and $\mathrm{Si} L_{2,3}$ (dashed lines) coherent fractions of the three SiC polytypes.

point are involved we conclude that while the states at the $X$ point do not have local $\mathrm{Si}(s+d)$ symmetry the states closer to the bottom of the LVB do so. However, the short lifetime of the final LVB hole state results in a strong broadening which makes any assignment difficult.

\section{Band dispersion of hexagonal $\mathrm{SiC}$}

In Fig. 9 we compare the coherent fractions of the spectra excited at threshold for the cubic and the hexagonal polytypes. One finds that the same energy range of the VB is involved in the coherent scattering in all polytypes for the $\mathrm{C}$ $K$ and Si $L_{2,3}$ spectra. The shape of the spectra, however, is different for the three polytypes. This demonstrates the increased sensitivity of RIXS compared to conventional SXE: As only a single $k$ point of the $\mathrm{BZ}$ is reached at threshold (or for higher energies a slice through momentum space) RIXS is sensitive to the small differences in the electronic structure of the different polytypes resulting from their different crystalline long range order. In the nonresonant SXE spectra these differences are washed out by integrating over the whole BZ.

The strongest difference is observed between the spectra of cubic $\mathrm{SiC}$ on one side and the hexagonal polytypes on the other. For $3 \mathrm{C}$-SiC only a single band contributes for excitation at each of the two thresholds while in the case of the hexagonal polytypes a manifold of bands is involved when scattering at threshold. These bands overlap in the spectra forming broader structures.

The increased number of bands is a result of the reduction in symmetry as the increased size of the unit cell results in a smaller BZ, i.e., the basis vectors of the BZ have a smaller absolute value. The "backfolding" of the bands results in a higher number of electron bands in the reduced band structure scheme. Consequently, there is a higher number of states at each point of the BZ, i.e., the number of states which fulfill the momentum condition $\mathbf{k}_{e}+\mathbf{k}_{h}+\mathbf{G}=\mathbf{0}$, with $\mathbf{G}$ a vector of the reciprocal lattice, increases with increasing size of the unit cell. All these states can contribute to the scattering when selecting the crystal momentum of the CBM by exciting at threshold as they all have the same momentum as the CBM (modulo a reciprocal lattice vector).

The CBM in the BZ of the hexagonal polytypes is located at the $M$ point. In $6 H$-SiC, however, the lowest energy band at the $L$ point is energetically very close to the CBM which gives rise to a larger momentum uncertainty (see Ref. 20). It is interesting to note that nevertheless the features in the spectra of $4 \mathrm{H}-\mathrm{SiC}$ are slightly broader than in $6 \mathrm{H}-\mathrm{SiC}$, which is in line with the calculations reproduced in Figs. 5-8. These predict the energy spread of the bands at the $M$ point to be larger in $4 H$ - than in $6 H-\mathrm{SiC}$.

Despite the manifold of states overlapping in the spectra, one can also find some distinct differences between the two hexagonal polytypes. The peak close to $-4 \mathrm{eV}$ in the $\mathrm{C} K$ spectra of $6 H$ (solid line at the top of Fig. 9) can be assigned to the "knot" of bands close to this binding energy between the $M$ and $L$ point in the calculation (see Fig. 5). For $4 H$ the calculation in Fig. 5 predicts a region of increased number of states at the $M$ point below $-2 \mathrm{eV}$ which is reflected by the intensity maximum in the $\mathrm{C} K$ threshold spectra (solid line in the middle of Fig. 9).

In the energy region of the LVB one finds weak intensity in the $\mathrm{Si}$ threshold spectra of both hexagonal polytypes in contrast to the case of $3 C-\mathrm{SiC}$. This is also due to the larger period with the consequence that more states can fulfill the momentum relation. Thereby, states with local $\mathrm{Si}(s+d)$ symmetry are backfolded to the momentum of the CBM in the hexagonal band structure (the "corresponding" states in cubic $\mathrm{SiC}$ are not located at the $k$ point of the CBM in a reduced band structure scheme).

We now turn our attention to the excitation energy dependence of the coherent fractions plotted for the hexagonal polytypes in Figs. 5-8. As in the case of cubic SiC (Ref. 20) we observe dispersion of emission lines and emission features appearing and disappearing. However, these effects are much less clear than in cubic SiC due to the increased number of bands in the BZ. This shows a general experimental difficulty common to all techniques when investigating the band structure of materials with large unit cells.

First we focus on the Si $L_{2,3}$ spectra which are dominated by a peak close to the bottom of the UVB. With increasing excitation energy the high-energy part of the UVB becomes more intense relative to the main peak. Especially, one observes that the high-energy cutoff of the spectral intensity distribution in the coherent fractions shifts towards the VBM. This reflects the general upward dispersion of the occupied bands when going towards the $\Gamma$ point. While in the case of $4 H-\mathrm{SiC}$ faint discrete peaks can still be distinguished in certain spectra, no discrete lines are resolvable in the $6 \mathrm{H}$ $\mathrm{SiC}$ spectra, which is due to the increasing number of states at each $k$ point as discussed before.

One also notes that for certain higher excitation energies weak traces of intensity occur in the energy range of the LVB. Again, this is stronger for the hexagonal polytypes than for cubic $\mathrm{SiC}$.

For the spectra excited above the $\mathrm{C} K$ edge shown in Fig. 5 and Fig. 7 one observes the same general trend in the coherent fractions, the high-energy cutoff moving towards the VBM with increasing excitation energy. Additionally, 


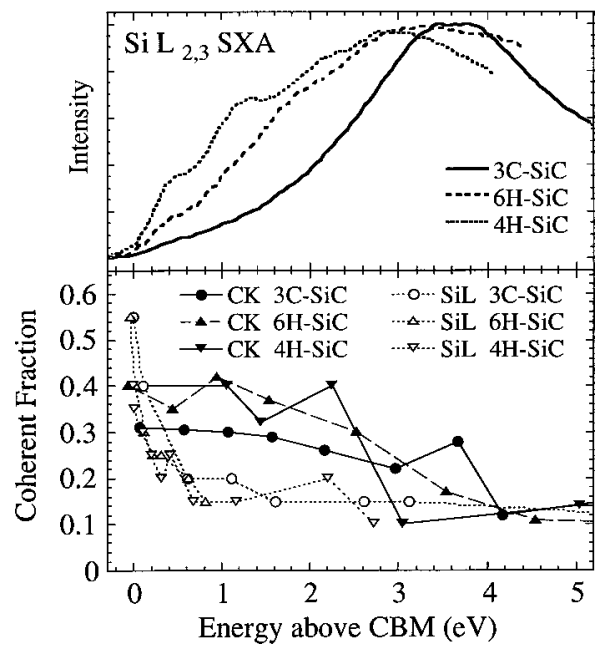

FIG. 10. Comparison for the different polytypes of the excitation energy dependence of the fraction by which coherent scattering contributes to the measured spectra (percentage values from Figs. $5-8$, and from Ref. 20). The uncertainty of these values is around $\pm 5 \%$. The $\mathrm{Si} L_{2,3}$ SXA spectra are plotted on the same energy scale.

one notices that even for such high excitation energies reaching the $\Gamma$ point of the $\mathrm{BZ}$ a coherent fraction is observed at the C $K$ edge. This is in contrast to the case of the $\mathrm{Si} L_{2,3}$ edge where the coherent fraction drops drastically within 1 $\mathrm{eV}$ above the absorption threshold. The different behavior can be understood by taking into account that the ratio of the core hole lifetime and the electron scattering time relates to the amount of the incoherent contribution to the scattering spectra. As the lifetime of a $\mathrm{C} 1 s$ core hole is shorter than that of a Si $2 p$ core hole ${ }^{36}$ the incoherent contribution is expected to be lower at the $\mathrm{C} K$ edge. Consequently, at the $\mathrm{C}$ $K$ edge a coherent fraction can be observed for higher excitation energies.

For excitation energies involving the $\Gamma$ point the high number of states at the VBM gives rise to a strong discrete emission peak at the high-energy side of the coherent $\mathrm{C} K$ spectra. However, due to the multitude of close-lying bands it is not possible to unambiguously assign the emission to single states.

The general upward trend of the high-energy cutoff in the coherent spectra with increasing excitation energy for all polytypes under investigation shows that all these polytypes are indirect band gap materials. This result follows from the experimental spectra alone without any assumption about the dispersion of the $\mathrm{CB}$ as in a direct band gap material the highest-energy emission would be observed for excitation at threshold. The indirect nature of the band gap can also be seen directly in the measured SXE spectra given by the dashed lines in Figs. 5-8, i.e., this observation is independent of the determination of the incoherent fraction.

\section{E. Energy dependence of coherent contribution}

In Fig. 10 the excitation energy dependence of the amount which the coherent fractions give to the measured spectra are shown for the three polytypes (percentage values from Figs. $5-8$, and from Ref. 20). The common energy scale is given by the energy of the excited electron in the CB. In addition, the Si $L_{2,3}$ absorption spectra are plotted on the same energy scale. One observes that the contribution of the coherent fraction decreases with increasing energy of the CB electron. This is consistent with the assumption that electron-phonon and electron-electron scattering in the intermediate state lead to the incoherent contribution of the spectra. Both scattering rates generally increase with increasing excess energy due to the increasing phase space available for scattering. ${ }^{39}$ The figure also illustrates how the resonantly excited spectra evolve with increasing excitation energy into the one determined by the LPDOS as mapped by the nonresonantly excited SXE spectrum. The variation with the excitation energy is large close to threshold, and the LPDOS is approached asymptotically at higher energies.

Comparing the $\mathrm{Si} L_{2,3}$ data to those from the C $K$ edge one observes that (for all polytypes) the coherent contribution decreases more rapidly at the Si $L_{2,3}$ edge. Considering the longer lifetime of a $\mathrm{Si} 2 p$ core hole excitation, ${ }^{36}$ the sharper decrease of the coherent fraction reflects the increasing time available for the violation of the momentum relation.

One further notices that the contribution of the coherent fractions to the at threshold excited spectra is lower in the case of the C $K$ spectra. These differences only slightly exceed the uncertainty, but they may indicate that for excitation of a $\mathrm{C} 1 s$ core hole the coupling to phonons is stronger than for the excitation of a Si $2 p$ core hole. This observation is supported by the weak ionic character of the bonds in $\mathrm{SiC}$. As carbon has a stronger electronegativity than silicon the bonding in $\mathrm{SiC}$ is more similar to the one in III- $\mathrm{V}$ compounds as compared to other IV-IV compounds, e.g., $\mathrm{SiGe}^{40}{ }^{4}$ The unoccupied states are thus slightly polarized towards the $\mathrm{Si}$ atoms, which may give rise to a stronger coupling to phonons for a $\mathrm{C} 1 s$ core hole excitation.

According to the phase space argument given above one would expect that the shape of the DOS at the bottom of the CB should have an influence on the decrease of the coherent fraction. The absorption spectra showing a sharper increase of the LPDOS at the CBM going from $3 \mathrm{C}$ via $6 \mathrm{H}$ to $4 \mathrm{H}$ suggest that the evolution of the contribution should be different for the three polytypes, namely, the decrease of the contribution of the coherent fraction should be stronger in $4 H$ than in $4 H$ than in $3 C$. However, such differences cannot be resolved within the experimental uncertainty of this study.

\section{CONCLUSIONS}

The electronic structure of $3 \mathrm{C}-, 6 \mathrm{H}-$, and $4 \mathrm{H}$-SiC has been investigated by nonresonantly and resonantly excited SXE spectroscopy. Due to the bulk sensitivity of this photon-in photon-out technique the measurement is not influenced by surface reconstructions or contaminations.

With nonresonant SXE spectroscopy the local $\mathrm{Si}(s+d)$ and $\mathrm{C} p$-symmetric DOSs have been experimentally determined. The occupied LPDOS for the polytypes under investigation is very similar, in particular, the VBM is located at the same emission energy within the experimental uncertainty of $100 \mathrm{meV}$. The opening of the band gap from $2.2 \mathrm{eV}$ for $3 C-\mathrm{SiC}$ to $3.3 \mathrm{eV}$ for $4 H-\mathrm{SiC}$ results from changes in the CB. The upward shift of the CBM is accompanied by 
changes in the unoccupied LPDOS, especially, an increase of the DOS close to the CBM.

Information about the band dispersion is obtained by resonantly excited SXE spectroscopy which is used as a tool for bulk sensitive and element specific band mapping resolving the local symmetry of the band states. The comparison of the threshold excited spectra highlights the differences in the occupied part of the band structure of the three polytypes.
*Present address: IBM Almaden Research Center, 650 Harry Road, San Jose, CA 95120.

†Present address: Department of Physics, Uppsala University, Box 530, 75121 Uppsala, Sweden.

${ }^{1}$ G. Pensl and R. Helbig, in Silicon Carbide (SiC) - Recent Results in Physics and in Technology, Festkörperprobleme/ Advances in Solid State Physics Vol. 30, edited by U. Rössler (Vieweg, Braunschweig, 1990).

${ }^{2}$ Z. Sitar, L.L. Smith, and R.F. Davis, J. Cryst. Growth 141, 11 (1994).

${ }^{3}$ J. Schardt, Ch. Bram, S. Müller, U. Starke, K. Heinz, and K. Müller, Surf. Sci. 337, 232 (1995).

${ }^{4}$ L.S. Ramsdell, Am. Mineral. 32, 64 (1947).

${ }^{5}$ H. Jagodzinski, Acta Crystallogr. 2, 201 (1949); R.W.G. Wyckoff, Crystal Structures, 2nd ed. (R.E. Krieger Publishing Company, Malabar, 1982), Vol. 1.

${ }^{6}$ CREE Research Inc., 2810 Meridian Parkway, Durham, NC 27713.

${ }^{7}$ G. Wiech, Z. Phys. 207, 428 (1967).

${ }^{8}$ I.I. Zhukova, V.A. Fomichev, A.S. Vinogradov, and T.M. Zimkina, Fiz. Tverd. Tela (Leningrad) 10, 1383 (1968) [Sov. Phys. Solid State 10, 1097 (1968)].

${ }^{9}$ M.L. Shek, K.E. Miyano, Q.-Y. Dong, T.A. Callcott, and D. Ederer, J. Vac. Sci. Technol. A 12, 1079 (1994).

${ }^{10}$ C.U.S. Larsson, A. Beutler, O. Björneholm, F. Federmann, U. Hahn, A. Rieck, S. Verbin, and T. Möller, Nucl. Instrum. Methods Phys. Res. A 337, 603 (1994).

${ }^{11}$ W. Eberhardt, G. Kalkoffen, C. Kunz, D. Aspnes, M. Cardona, Phys. Status Solidi B 88, 135 (1978).

${ }^{12}$ J. Stöhr, in NEXAFS Spectroscopy, 2nd ed., Springer Series in Surface Science, Vol. 25 (Springer, Heidelberg, 1992).

${ }^{13}$ S. Eisebitt, T. Böske, J.-E. Rubensson, and W. Eberhardt, Phys. Rev. B 47, 14103 (1993).

${ }^{14}$ H. Hoechst, M. Tang, B.C. Johnson, J.M. Meese, G.W. Zajac, and T.H. Fleisch, J. Vac. Sci. Technol. A 5, 1640 (1987).

${ }^{15}$ L.I. Johansson, F. Owman, and P. Mårtensson, Phys. Rev. B 53, 13793 (1996).

${ }^{16}$ D.A. Goodings and R. Harris, J. Phys. C 2, 1808 (1969).

${ }^{17}$ U. von Barth and G. Grossmann, Phys. Rev. B 25, 5150 (1982).

${ }^{18}$ The PES spectrum was recorded at the undulator beamline X1B (Ref. 19) at the NSLS, Brookhaven, with a Scienta SES-200 spherical analyzer. A photon energy of $340 \mathrm{eV}$ was chosen for excitation to increase the contribution of bulk states to the spectra. The same SiC samples as in SXE were studied in normal emission, and the results are discussed in detail by L. Baumgarten, J. Lüning, S. Cramm, B. Kessler, A. Scholl, and W. Eberhardt (unpublished).

${ }^{19}$ W. Eberhardt, K.J. Randall, J. Feldhaus, A. Bradshaw, R.F. Garrett, and M.L. Knotek, Phys. Scr. 41, 745 (1990).

${ }^{20}$ J. Lüning, J.-E. Rubensson, C. Ellmers, S. Eisebitt, and W. Eberhardt, Phys. Rev. B 56, 13147 (1997).

${ }^{21}$ K. Siegbahn, C. Nordling, G. Johansson, J. Hedman, P. F. Heden, K. Hamrin, U. Yelius, T. Bergmark, L. O. Werme, R. Manne, and Y. Baer, ESCA Applied to Free Molecules (North-Holland, Amsterdam, 1969).

${ }^{22}$ Practical Surface Analysis, Vol. 1: Auger and X-Ray Photoelectron Spectroscopy, 2nd ed., edited by D. Briggs and M.P. Seah (Wiley, Chichester, 1990).

${ }^{23}$ C.H. Park, B.-H. Cheong, K.-H. Lee, and K.J. Chang, Phys. Rev. B 49, 4485 (1994).

${ }^{24}$ P. Käckell, B. Wenzien, and F. Bechstedt, Phys. Rev. B 50, 10761 (1994).

${ }^{25}$ N. Wassdahl, J.-E. Rubensson, G. Bray, P. Glans, P. Bleckert, R. Nyholm, S. Cramm, N. Mårtensson, and J. Nordgren, Phys. Rev. B 64, 2807 (1990).

${ }^{26}$ J.-E. Rubensson, D. Mueller, R. Shuker, D.L. Ederer, C.H. Zhang, J. Jia, and T.A. Callcott, Phys. Rev. Lett. 64, 1047 (1990).

${ }^{27}$ Y. Ma, N. Wassdahl, P. Skytt, J. Guo, J. Nordgren, P.D. Johnson, J.-E. Rubensson, T. Böske, W. Eberhardt, and S.D. Kevan, Phys. Rev. Lett. 69, 2598 (1992).

${ }^{28}$ F. Gel'mukhanov, L.N. Mazalov, and N.A. Shklyaeva, Zh. Eksp. Teor. Fiz 71, 960 (1976) [Sov. Phys. JETP 44, 504 (1976)].

${ }^{29}$ J.A. Carlisle, E.L. Shirley, E.A. Hudson, L.J. Terminello, T.A. Callcott, J.J. Jia, D.L. Ederer, R.C.C. Perera, and F.J. Himpsel, Phys. Rev. Lett. 74, 1234 (1995).

${ }^{30}$ A. Agui, S. Shin, M. Fujisawa, Y. Tezuka, T. Ishii, O. Mishima, K. Era, E. Shigemasa, and A. Yagishita, J. Electron. Spectrosc. Relat. Phenom. 79, 191 (1996).

${ }^{31}$ K.E. Miyano, D.L. Ederer, T.A. Callcott, W.L. O’Brien, J.J. Jia, L. Zhou, Q.-Y. Dong, Y. Ma, J.C. Woicik, and D.R. Mueller, Phys. Rev. B 48, 1918 (1993).

${ }^{32}$ S. Eisebitt, J. Lüning, J.-E. Rubensson, A. Settles, P. H. Dedirichs, W. Eberhardt, S. N. Patitsas, and T. Tiedje, J. Electron Spectrosc. Relat. Phenom. 93, 245 (1998).

${ }^{33}$ For a discussion of the validity of this interpretation see Ref. 20 , and references therein.

${ }^{34}$ M. van Veenendaal and P. Carra, Phys. Rev. Lett. 78, 2839 (1997), and references therein.

${ }^{35}$ The validity of this assumption is demonstrated experimentally for the case of several broadband materials, e.g., Refs. 20, 26, 27 , and $29-31$.

${ }^{36}$ The lifetime broadening of a Si $2 p$ core hole state as measured in PES on $\mathrm{SiH}_{4}$ is $45 \mathrm{meV}$ (Ref. 37) while for a C $1 s$ core hole in $\mathrm{CH}_{4}$ the broadening is determined to be $83 \mathrm{meV}$ (Ref. 38).

${ }^{37}$ J.D. Bozek, G.M. Bancroft, J.N. Cutler, and K.H. Tan, Phys. Rev. Lett. 65, 2757 (1990).

${ }^{38}$ H.M. Köppe, B.S. Itchkawitz, A.L.D. Kilcoyne, J. Feldhaus, B. Kempgens, A. Kivimäki, M. Neeb, and A.M. Bradshaw, Phys. Rev. A 53, 4120 (1996).

${ }^{39}$ In a forthcoming publication by S. Eisebitt, J. Lüning, J.-E. Rubensson, A. Karl, C. Ellmers, and W. Eberhardt, the importance of electron-phonon scattering for the origin of the incoherent fraction will be discussed in detail analyzing the results obtained for several semiconducting broadband materials.

${ }^{40}$ M. Willatzen, M. Cardona, and N.E. Christensen, Phys. Rev. B 51, 13150 (1995) 